\title{
Resolution Measured as Separability Compared to Full Width Half Maximum for Adaptive Beamformers
}

\author{
*Department of Informatics \\ University of Oslo \\ Oslo, Norway \\ Email: omrindal@ifi.uio.no
}

Ole Marius Hoel Rindal*, Alfonso Rodriguez-Molares ${ }^{\dagger}$

and Andreas Austeng*

\begin{abstract}
Spatial resolution is defined as a system's ability to separate targets using some kind of criterion, such as the Rayleigh criterion. However, in practice, lateral resolution is often evaluated by measuring the Full Width Half Maximum (FWHM at -6dB) of the point spread function (PSF). We hypothesize that FWHM overestimates the system resolution for some adaptive beamformers, compared to using the Rayleigh criterion. Simulation results seem to confirm this hypothesis.

Index Terms-Imaging metrics, resolution, adaptive beamforming.
\end{abstract}

\section{INTRODUCTION}

The Full Width Half Maximum (FWHM) is the most commonly used metric when evaluating the resolution of an ultrasound imaging system or an ultrasound beamforming algorithm. FWHM is measured as the width of the mainlobe in the point spread function (PSF), or beampattern, at half amplitude in linear scale, or at $-6 \mathrm{~dB}$ in decibel scale. Intuitively, smaller FWHM values should mean improved separability, i.e. better separation between closely placed targets. We hypothesise that some adaptive beamforming algorithms may decrease the FWHM without increasing target separability. Some authors have included an analysis of separability when evaluating beamforming algorithms [1] [2] [3]. However, the consequences of using FWHM as a measure of resolution for adaptive beamformers could lead to the wrong conclusions. This needs to be brought to attention to the ultrasound community. This paper is structured as follows, in Section II we will review the FWHM as a resolution metric using theoretical examples of ultrasound arrays, Section III describe the data and methods used to produce the results in Section IV followed by a discussion in Section V and conclusion in Section VI. Parts of the results have been presented in the background chapter of [4].

\section{BACKGROUND}

A. The Full Width Half Maximum (FWHM) of an ultrasound array

The expected spatial resolution of a transducer array, is often calculated by estimating the FWHM of the theoretical beam-

\author{
${ }^{\dagger}$ Defense University Center \\ University of Vigo \\ Marin, Spain
}

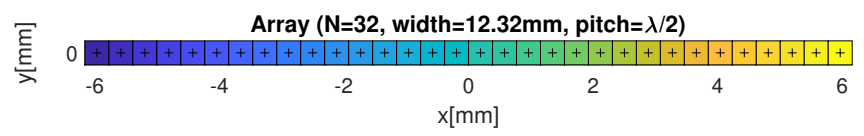

(a) Array Geometry

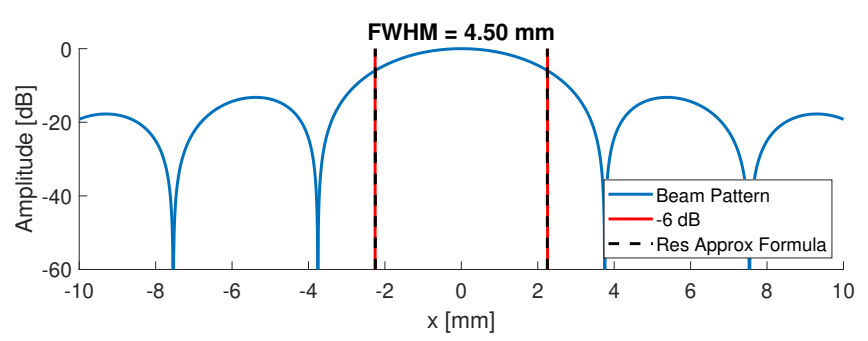

(b) Beampattern

Fig. 1: The array geometry (a) of a $\mathrm{N}=32, \frac{\lambda}{2}$ pitch array with the resulting beampattern (b) estimated using eq. (1) with the $-6 \mathrm{~dB}$ limit indicated in red. The dashed black lines indicate the $-6 \mathrm{~dB}$ resolution as calculated by eq. (2).

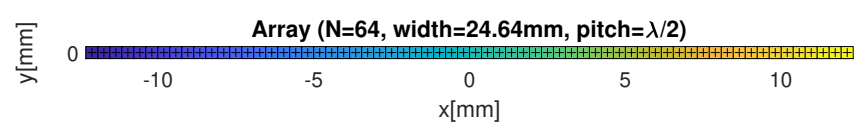

(a) $4 \mathrm{~mm}$ distance between scatterers

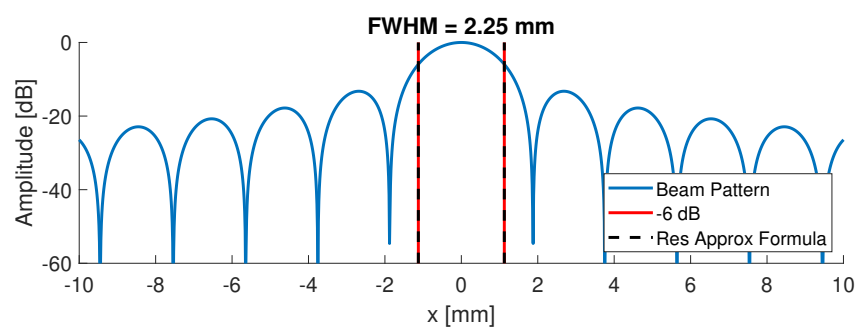

(b) $2 \mathrm{~mm}$ distance between scatterers

Fig. 2: The array geometry (a) of a $\mathrm{N}=64, \frac{\lambda}{2}$ pitch array with the resulting beampattern (b) estimated using eq. (1) with the $-6 \mathrm{~dB}$ limit indicated in red. The dashed black lines indicate the $-6 \mathrm{~dB}$ resolution as calculated by eq. (2). 
pattern. The one-way far-field single frequency beampattern is given by the discrete aperture smoothing function, the discrete Fourier transform, of an array along the $\mathrm{x}$-axis as, see e.g., [5]

$$
W\left(k_{x}\right)=\sum_{m=0}^{N-1} w_{m} e^{j k_{x} x_{m}} .
$$

Here $w_{m}$ is the element weight, $k_{x}$ is the $x$ component of the wavenumber vector, and $x_{m}$ is the element position. The relation between $k_{x}$ and the incidence angle $\theta$ of the wave is, $k_{x}=-k \sin \theta=-\frac{2 \pi}{\lambda} \sin \theta$ [5]. The far-field beampattern resulting from eq. (1) is a good approximation of the beampattern in focus for a finite bandwidth in the nearfield [6].

If we have an ultrasound array with geometry as in Fig. 1a with $N=32$ elements, a pitch of $\lambda / 2$, assume a speed of sound at $1540 \mathrm{~m} / \mathrm{s}$ and a narrow band with center frequency at $f_{c}=2 \mathrm{MHz}$ and we can use eq. (1) and get the one-way beampattern at range $R=60 \mathrm{~mm}$ in Fig. 1b.

Similarly, if we use the same parameters, but extend the array to $N=64$ elements we get the results in Fig. 2 .

In both Fig. $1 \mathrm{~b}$ and $2 \mathrm{~b}$ we indicated the resulting resolution measured as the FWHM by estimating the width of the beampattern at $-6 \mathrm{~dB}$ as well as using the well known approximation formula for the angular resolution at $-6 \mathrm{~dB}$ [7]:

$$
\theta_{6 d B} \approx \frac{1.21 \lambda}{D}
$$

where $D$ is the size of the full array aperture. Notice how the resolution is inverse proportional to the size of the array, and proportional to the signal frequency through the wavelength $\lambda$. The spatial lateral resolution at $R$, can be found using a small angle approximation.

When comparing the resulting FWHM from the two examples, we see that the resolution of the array with $N=64$ elements have improved with a factor of 2 compared to the array of $N=32$, thus from a FWHM of $4.50 \mathrm{~mm}$ to 2.25 $\mathrm{mm}$ assuming both arrays transmitted a focused wave with focal point in $R=60 \mathrm{~mm}$, which is in agreement with eq. (2). Thus, the FWHM is a great metric for a linear system assuming conventional Delay-And-Sum (DAS) beamforming with known weights $w$.

\section{B. Resolution measured as separability}

Multiple definitions for metrics to measure the resolution as separability exists. Among them are Sparrow's resolution limit, defined as where the saddle point between the two peaks first develops. Rayleigh's resolution criterion defines two points as separated if the minimum between them are more than a certain threshold. According to the Rayleigh criterion, we consider two targets as separated if there is a pixel between them where the signal intensity has dropped by at least $6 \mathrm{~dB}$.

\section{METHODS}

We simulated point scatterers in Field II[8][9], mimicking a L7-4 linear array with 128 elements transmitting a single plane wave at $5.16 \mathrm{MHz}$. The channel data was beamformed in MATLAB (The MathWorks, Natick, USA) using the UltraSound ToolBox [10] using 7 different beamforming methods:

1) delay-and-sum (DAS),

2) Mallart-Fink's coherence factor (CF) [11],

3) Camacho et al.'s phase coherence factor (PCF) [3],

4) Li-Li's generalized coherence factor (GCF) [12],

5) Capon's minimum variance beamformer (MV) [13],

6) the Eigenspace Based Minimum Variance (EBMV) [14],

7) Matrone et al.'s filtered delay-multiply-and-sum (FDMAS) [15].

For more details on the implementations used see the original references, or [16]. To evaluate the FWHM we simulated a single point scatter and measured the lateral distance at $-6 \mathrm{~dB}$. To evaluate the separability, we imaged two point scatterers separated by $4,2,1,0.5,0.44$ and $0.4 \mathrm{~mm}$. Since the plane wave transmission is unfocused the receive beamforming
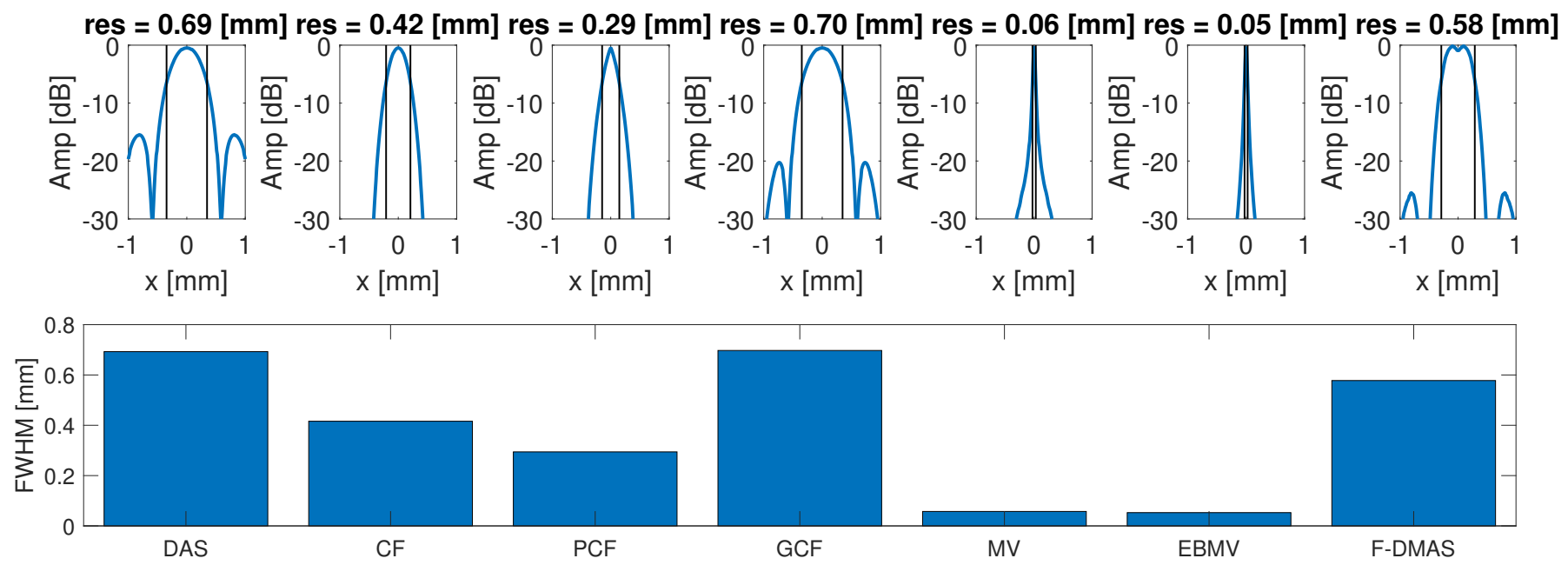

Fig. 3: The estimated FWHM for all beamformers under test. The top plot displays the mainlobe of the beampattern with the resolution measured as FWHM indicated in the title. The resulting FWHM values are plotted in the bar graph below. 
results in a one-way PSF corresponding to the one-way beampattern from eq. (1).

\section{RESUlts}

The top row in Fig. 3 is the mainlobe of the PSFs for all beamformers when imaging a single point scatterer. The FWHM is indicated in the title above and in the bars below. Judging by the results in Fig. 3 all the adaptive beamformers tested, except GCF, show better lateral resolution than DAS. Fig. 4a to $4 \mathrm{f}$ are the resulting images of two point scatterers separated from 4 to $0.4 \mathrm{~mm}$. The red dashed line in the plot marks the $-6 \mathrm{~dB}$ value. In Fig. 5a we display the measured separability, in $\mathrm{dB}$, from the maximum of the point scatters to the minimum between them. In Fig. $5 \mathrm{~b}$ we have thresholded on the $-6 \mathrm{~dB}$ limit, into separated or not separated. With $1 \mathrm{~mm}$ or higher separation, all the beamformers expect GCF are able to detect both scatterers, with detection defined as a negative peak of more than $-6 \mathrm{~dB}$ between the points. At $0.5 \mathrm{~mm}$ separation only MV and EBMV can separate the scatterers. Finally, at $0.4 \mathrm{~mm}$ and $0.44 \mathrm{~mm}$ only $\mathrm{MV}$ is able to separate the two scatterers.

\section{DISCUSSION}

Interestingly, only the MV and EBMV beamformer have better target separability than DAS. Even though the FWHM indicated that all beamformers except GCF improved the FWHM compared to DAS. The results seem to confirm our hypothesis that FWHM overestimates the resolution limit for some beamformers compared to a metric based on target separability. Further work is needed to derive a criteria measuring separability, whose results are independent of non-linear operations from some adaptive beamformers, and that can be used in targets of different strength. Such a criterion is of importance to assess the relevance of new beamforming techniques. New metrics have been shown to be important to correctly evaluate the contrast of adaptive beamformers [16] [17] [18]. We believe the presented results show this is relevant for resolution metrics as well.

\section{CONCLUSION}

The conventional full width half maximum (FWHM) and a separability measure have been evaluated as metrics for lateral resolution. The results show that some adaptive beamformers only improve the resolution as measured by FWHM while not improving the separability of point targets as measured using the Rayleigh criterion. This puts into question the relevance of FWHM as a measure for lateral resolution.

\section{ACKNOWLEDGEMENTS}

This work was supported in part by the Center for Innovative Ultrasound Solutions (CIUS) and the Research Council of Norway under Project 237887.

\section{REFERENCES}

[1] K. Diamantis, T. Anderson, M. B. Butler, C. A. Villagomez-Hoyos, J. A Jensen, and V. Sboros, "Resolving ultrasound contrast microbubbles using minimum variance beamforming," IEEE Transactions on Medical Imaging, vol. 38, no. 1, pp. 194-204, 2019.

[2] A. C. Jensen and A. Austeng, "The iterative adaptive approach in medical ultrasound imaging." IEEE transactions on ultrasonics, ferroelectrics, and frequency control, vol. 61, no. 10, pp. 1688-97, 2014 [Online]. Available: http://www.ncbi.nlm.nih.gov/pubmed/25265177

[3] J. Camacho, M. Parrilla, and C. Fritsch, "Phase coherence imaging," IEEE Transactions on Ultrasonics, Ferroelectrics, and Frequency Control, vol. 56, no. 5, pp. 958-974, 2009.

[4] O. M. H. Rindal, "Software Beamforming in Medical Ultrasound Imaging - a blessing and a curse Thesis submitted for the degree of Philosophiae Doctor," Ph.D. dissertation, University of Oslo, 2019.

[5] D. H. Johnson and D. E. Dugdeon, Array signal processing: Concepts and techniques. UpperSaddle River, NJ 07458: P.T.R. Prentice-Hall, 1993, vol. Prentice-Hall signal processing series.

[6] B. D. Steinberg, Principles of Aperture and Array System Design. John Wiley \& Sons, 1976.

[7] F. J. Harris, "On the Use of Windows for Harmonic Analysis with the Discrete Fourier Transform," Proceedings of the IEEE, vol. 66, no. 1, pp. 51-83, 1978.

[8] J. A. Jensen and N. B. Svendsen, "Calculation of Pressure Fields from Arbitrarily Shaped, Apodized, and Excited Ultrasound Transducers," IEEE Transactions on Ultrasonics, Ferroelectrics and Frequency Control, vol. 39, no. 2, pp. 262-267, 1992.

[9] J. A. Jensen, "Field: A program for simulating ultrasound systems," Medical \& Biological Engineering \& Computing, vol. 34, pp. 351-353, 1996.

[10] A. Rodriguez-Molares, O. M. H. Rindal, O. Bernard, A. Nair, M. A. Lediju Bell, H. Liebgott, A. Austeng, and L. Løvstakken, "The UltraSound ToolBox," IEEE International Ultrasonics Symposium, IUS, pp. 1-4, 2017.

[11] R. Mallart and M. Fink, "Adaptive focusing in scattering media through sound-speed inhomogeneities: The van Cittert Zernike approach and focusing criterion," The Journal of the Acoustical Society of America, vol. 96, no. 6, p. 3721, 1994.

[12] P. C. Li and M. L. Li, "Adaptive imaging using the generalized coherence factor," IEEE Transactions on Ultrasonics, Ferroelectrics, and Frequency Control, vol. 50, no. 2, pp. 128-141, 2003.

[13] J.-F. Synnevåg, A. Austeng, and S. Holm, "Benefits of minimumvariance beamforming in medical ultrasound imaging," Ultrasonics, Ferroelectrics and Frequency Control, IEEE Transactions on, vol. 56, no. 9, pp. 1868-1879, 2009.

[14] B. M. Asl and A. Mahloojifar, "Eigenspace-based minimum variance beamforming applied to medical ultrasound imaging," IEEE Transactions on Ultrasonics, Ferroelectrics, and Frequency Control, vol. 57, no. 11 , pp. 2381-2390, 2010

[15] G. Matrone, A. S. Savoia, G. Caliano, S. Member, and G. Magenes, "The Delay Multiply and Sum Beamforming Algorithm in Ultrasound B - Mode Medical Imaging," IEEE Trans. Med. Imaging, vol. 34, no. 4, pp. 1-10, 2015.

[16] O. M. H. Rindal, A. Austeng, A. Fatemi, and A. Rodriguez-Molares, "The Effect of Dynamic Range Alterations in the Estimation of Contrast," IEEE Transactions on Ultrasonics, Ferroelectrics, and Frequency Control, vol. 66, no. 7, pp. 1198-1208, 2019.

[17] O. M. H. Rindal, A. Austeng, H. Torp, S. Holm, and A. RodriguezMolares, "The dynamic range of adaptive beamformers," IEEE International Ultrasonics Symposium, IUS, no. 1, pp. 1-4, 2016.

[18] A. Rodriguez-Molares, O. M. H. Rindal, J. D’hooge, S.-E. Måsøy, A. Austeng, and H. Torp, "The Generalized Contrast-to-Noise Ratio," IEEE International Ultrasonics Symposium, IUS, no. 6, pp. 1-4, 2018. 

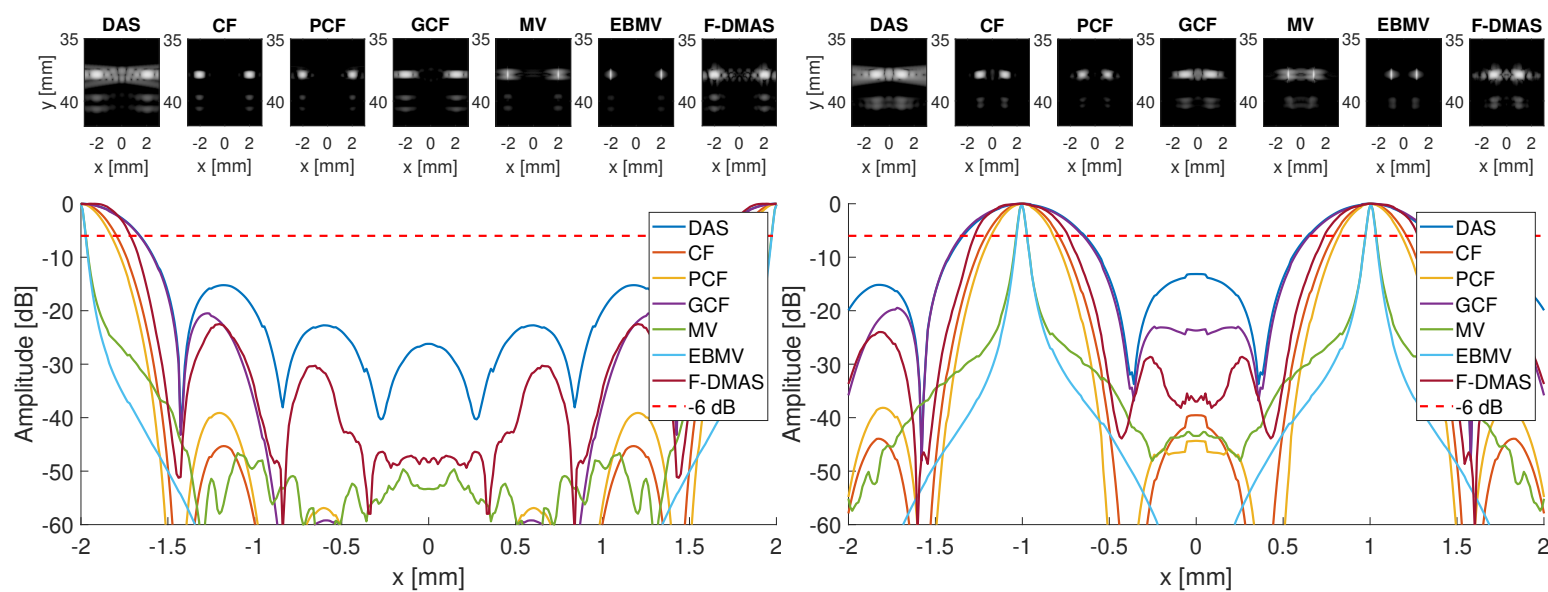

(a) $4 \mathrm{~mm}$ distance between scatterers
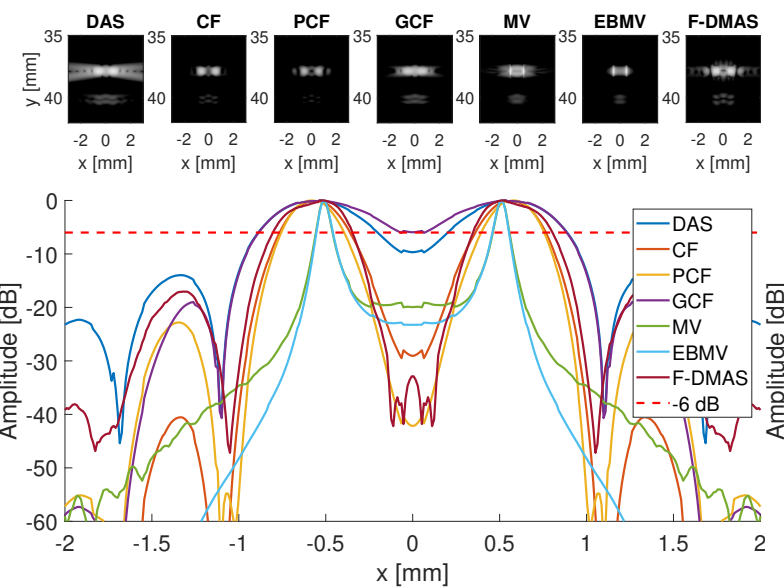

(c) $1 \mathrm{~mm}$ distance between scatterers

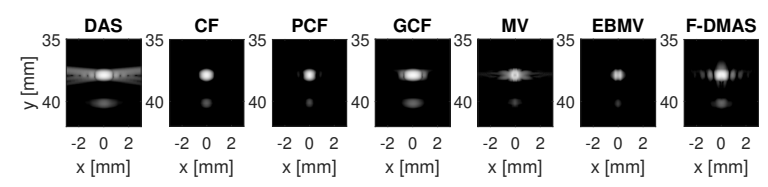

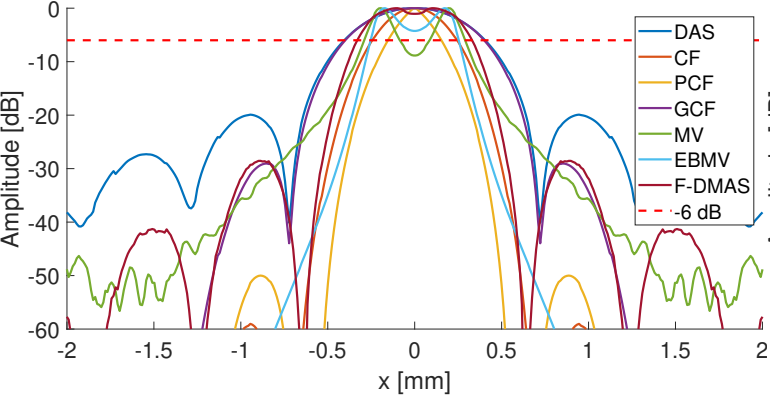

(e) $0.44 \mathrm{~mm}$ distance between scatterers

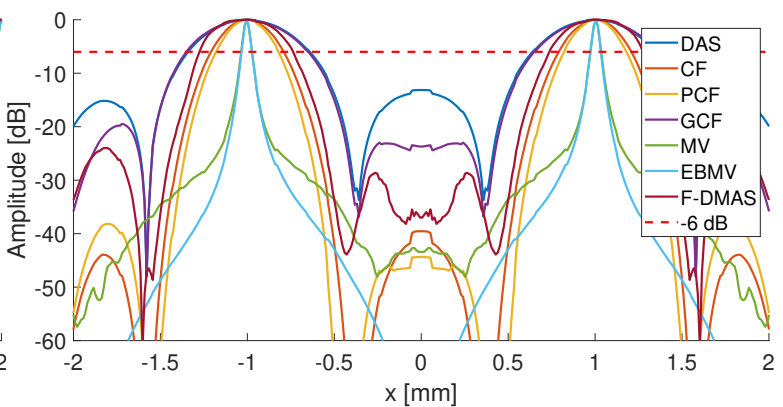

(b) $2 \mathrm{~mm}$ distance between scatterers
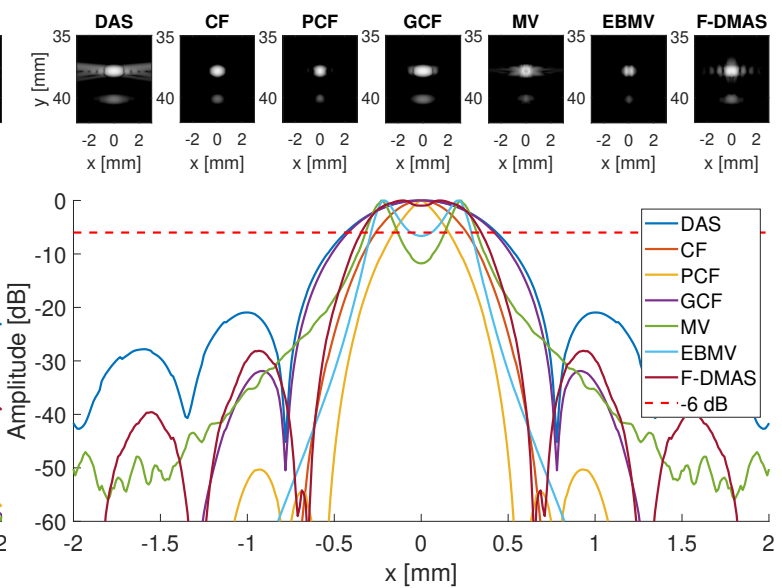

(d) $0.5 \mathrm{~mm}$ distance between scatterers

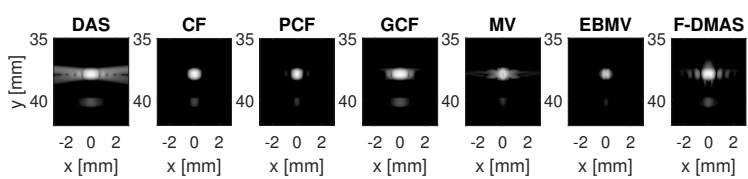

Fig. 4: Two point scatterers separated by 4, 2, 1, 0.5, 0.44 and $0.4 \mathrm{~mm}$ from (a) to (f)

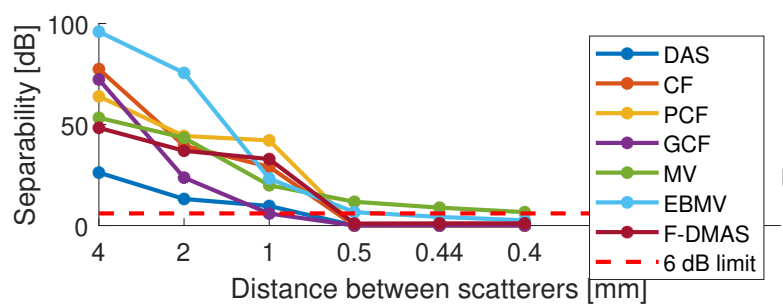

(a) Separability in $\mathrm{dB}$

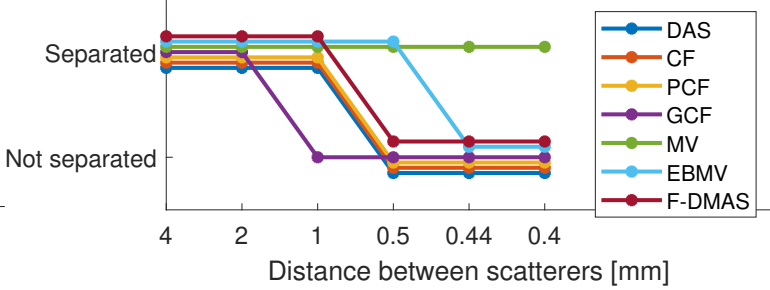

(b) Separability thresholded on $-6 \mathrm{~dB}$

Fig. 5: The measured separability, thus the minimum between the peaks, is plotted in (a), while the same values but thresholded into separated or not separated in (b). 